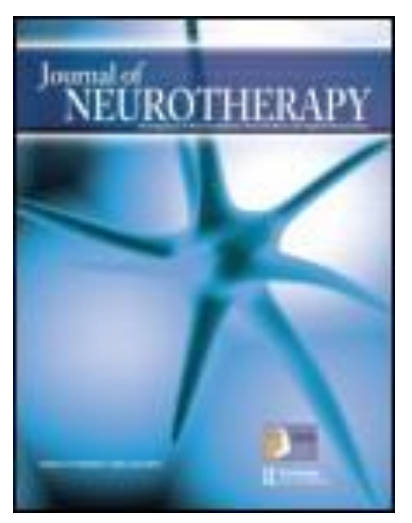

Journal of Neurotherapy: Investigations in
Neuromodulation, Neurofeedback and Applied
Neuroscience

SELECTED ABSTRACTS of CONFERENCE PRESENTATIONS

at the 2013 INTERNATIONAL SOCIETY for NEUROFEEDBACK \& RESEARCH (ISNR) 21ST ANNUAL CONFERENCE, DALLAS, TEXAS

Published online: 04 Dec 2013.

To cite this article: (2013) SELECTED ABSTRACTS of CONFERENCE PRESENTATIONS at the 2013 INTERNATIONAL SOCIETY for NEUROFEEDBACK \& RESEARCH (ISNR) 21ST ANNUAL CONFERENCE, DALLAS, TEXAS, Journal of Neurotherapy: Investigations in Neuromodulation, Neurofeedback and Applied Neuroscience, 17:4, 272-288, DOI: $\underline{10.1080 / 10874208.2013 .855484}$

To link to this article: http://dx.doi.org/10.1080/10874208.2013.855484

PLEASE SCROLL DOWN FOR ARTICLE

(C) International Society for Neurofeedback and Research (ISNR), all rights reserved. This article (the "Article") may be accessed online from ISNR at no charge. The Article may be viewed online, stored in electronic or physical form, or archived for research, teaching, and private study purposes. The Article may be archived in public libraries or university libraries at the direction of said public library or university library. Any other reproduction of the Article for redistribution, sale, resale, loan, sublicensing, systematic supply, or other distribution, including both physical and electronic reproduction for such purposes, is expressly forbidden. Preparing or reproducing derivative works of this article is expressly forbidden. ISNR makes no representation or warranty as to the accuracy or completeness of any content in the Article. From 1995 to 2013 the Journal of Neurotherapy was the official publication of ISNR (www. Isnr.org); on April 27, 2016 ISNR acquired the journal from Taylor \& Francis Group, LLC. In 2014, ISNR established its official open-access journal NeuroRegulation (ISSN: 2373-0587; www.neuroregulation.org).

THIS OPEN-ACCESS CONTENT MADE POSSIBLE BY THESE GENEROUS SPONSORS
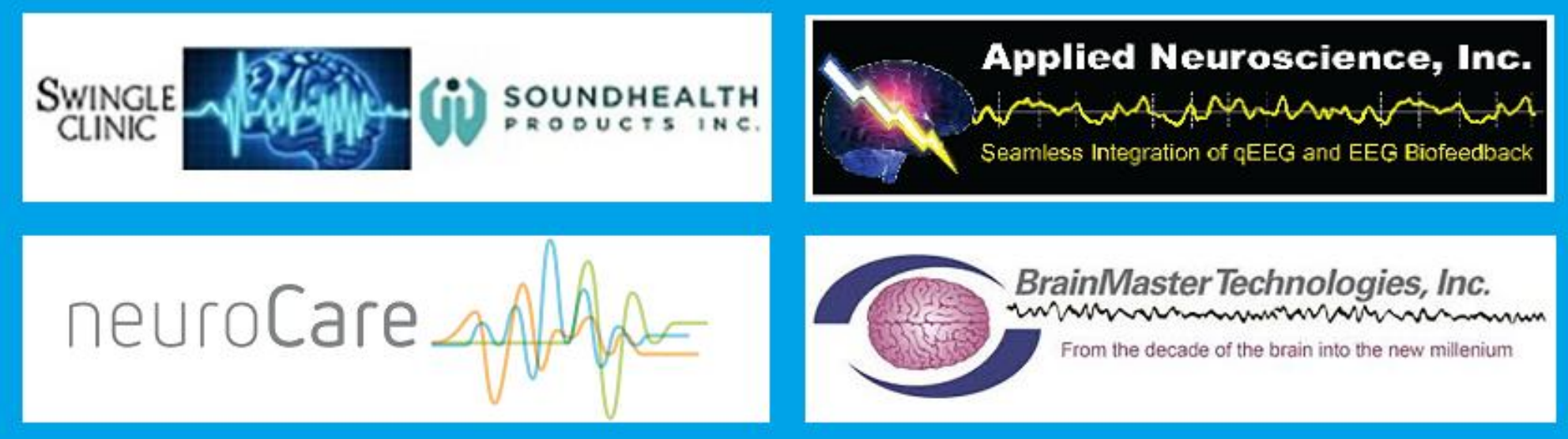


\section{PROCEEDINGS OF THE 2013 ISNR CONFERENCE SELECTED ABSTRACTS OF CONFERENCE PRESENTATIONS AT THE 2013
INTERNATIONAL SOCIETY FOR NEUROFEEDBACK \& RESEARCH (ISNR)
21ST ANNUAL CONFERENCE, DALLAS, TEXAS}

\section{Long-Term Follow-Up of Neurofeedback Outcomes in Clients with Asperger's Syndrome}

Tanushree Bhandari

ADD Centre and Biofeedback Institute of Toronto

\section{Introduction}

In 2010 a study was published indicating improvement in symptoms of Asperger's syndrome as a result of a combination of neurofeedback, biofeedback, and metacognitive training (Thompson et al., 2010). This study represented the largest chart review to date $(N=150)$ investigating improvements in symptoms of Asperger's syndrome following a course of neurofeedback training. The aforementioned study measured results immediately following training. The question that remains is, How are these clients doing today? The goal of the present study is to measure the long-term benefits of neurofeedback combined with biofeedback and metacognitive training by examining the current level of functioning of clients who completed training more than 2 years ago. The hypothesis, based on long-term follow-up of clients with attention deficit/hyperactivity disorder (Gani et al., 2009; Leung, 2012; Lubar, 1995) is that the improvements noted at posttesting will be maintained at follow-up, that is, after improvement from pretraining to posttraining, scores will be stable from posttraining to follow-up (results will not be significantly different).

\section{Method}

The participant sample consisted of 23 clients who had earlier been seen at the ADD Centre for neurofeedback training. The sample included
21 male and 2 female clients. There were several criteria that had to be met for inclusion in this study. First, all clients were assessed and trained at the ADD Centre. Second, all participants had symptoms of Asperger's syndrome or had received a diagnosis of Asperger's syndrome. Third, all clients received neurofeedback and biofeedback training combined with metacognitive strategies at the ADD Centre between 1997 and 2011. Strategies selected aimed to assist with academics and social skills. Fourth, all clients completed a minimum of forty $50-\mathrm{min}$ training sessions. Last, all clients completed training at the centre between 2 and 14 years ago. Mean follow-up time was 6.5 years. All clients were mailed a package that included (a) an invitation to participate in the study; (b) information outlining the purpose, procedures, and possible benefits and risks of participating in the study, as well an explanation of the confidentiality agreement and withdrawal policy relating to the study; (c) a consent form; (d) a set of four questionnaires (for adults - The ACQ, The Adult Self-Report Scale-VI Screener, DSM-IV Rating Scale, and the Wender-Utah Rating Scale; for children-Conner's Global Index-Parent Version, DSM-IV symptom list, ADD-Q for children, and the Australian Scale for Asperger's); and (e) a comment form. Participants were also invited to come in for free update testing on the Integrated Visual and Auditory Continuous Performance task and Test of Variables of Attention.

\section{Results}

Data were compared across two data points: posttraining and at follow-up (i.e., currentlevel-of-functioning). Mean values were compared at each point for all questionnaire data. Preliminary results demonstrate that 
scores on all questionnaires (ADD-Q, DSM-IV, Conner's and Australian Scale for Asperger's Syndrome) at follow-up did not significantly differ from posttraining scores, indicating that clients had not experienced regression in functioning. Comment forms filled out by parents contained anecdotal evidence of benefits noticed by participants and parents. "We believe the Biofeedback supported him through a difficult time. It has only (had) positive effects." "In answering the questions (questionnaires), I realized that many of the questions that related to behaviors my child experienced before are not present today. I believe that the training he went through at the ADD Centre helped tremendously." "Removing him from school, taking him to his training (ADD Centre) gave Ramon (name changed) a quality of life that before training was quite improbable and impossible." "Katherine (name changed) has continued to advance since her treatments at the ADD Centre. The ADD Centre helped Katherine wean herself off of medication and to this day she has not needed to return to the use of meds."

\section{Limitations}

Given the data collection method of a mail-out to previous clients, we were limited in the responses we received, as several clients did not live at the same address anymore. This is expected with any long-term follow-up study. There could well be a response bias in favor of families who were happy with the outcomes and therefore motivated to take the time to be part of the study. However, the responses received from clients also reflected those who were seeking support and therefore returned for an updated assessment. We recognize that the sample for this pilot study is small, and hence these results are only a preliminary reflection. We hope to continue to collect data and increase the sample for our study.

\section{Conclusion}

These results support our hypothesis that the positive gains achieved through neurofeedback training, for clients with symptoms of Asperger's syndrome, are maintained long term.

\section{Keep Them Coming Back: Interpersonal Techniques and Their Relationship to Client Adherence, Engagement, and Satisfaction}

Jonathon Larson, Thomas Cothran, Christopher Haak, Lindsay Sheehan, Katherine Kereszturi, Bethany Apa, Catherine Ryan, Robert Beedle, and Kelly O'Neill

Illinois Institute of Technology

Rigorous empirical research continues to demonstrate the efficacy of neurofeedback therapy (NFT) in peak performance training and the treatment of psychiatric and neurological disorders (Hammond, 2007; Yucha \& Montgomery, 2008). However, according to NFT practitioners, one of the barriers to successful functional outcomes is client adherence and ambivalence to ongoing treatment (Larson, Ryan, \& Baerentzen, 2010). NFT provides an effective intervention for improving self-regulation of brain activity, arousal, and reducing unwanted symptoms. However, the technology does not inherently contain components targeted at facilitating continued client engagement. Human factors play a significant role in client adherence, engagement, and satisfaction with therapy. Research shows that patient engagement and support can be improved significantly using specific interpersonal techniques (Beck, Daughtridge, \& Sloane, 2002; Zolnierek \& DiMatteo, 2009). Moving beyond simple friendliness, this workshop will survey empirical literature related to professional comportment and rapport, transtheoretical common factors of treatment outcomes, motivational interviewing, and verbal and nonverbal communication. This workshop will fluidly combine dialectic and experiential teaching strategies to elucidate evidence-based strategies for guiding clients from first contact through successful termination of therapy. It is intended to benefit practitioners from a wide range of expertise, from the novice technician to the experienced clinician. 
qEEG and Neurofeedback Diagnosis and Treatment for Sports Acquired Traumatic Brain Injury

Michael Linden

Attention Learning Centers

Jay Gunkelman

Brain Science International

Alan Strohmayer

Brain Strength Training

The use of neurofeedback is gaining interest in sport medicine and athletic organizations as training for peak performance and, more recently, for traumatic brain injury (TBI) and postconcussive syndromes (PCS) rehabilitation. In this symposium we explore the use of qEEG to help identify the deficits resulting from sports acquired TBI. We review and explore the use of the qEEG and other psychological performance tests to describe the TBI and how they can be used to determine the correct time to return to play (RTP). RTP is currently a highly controversial topic in sports medicine. We show the role of qEEG Guided NF protocols in the recovery from TBI and other PCS, especially headache. The presenters draw upon clinical case studies of TBI successfully treated using these techniques.

\section{An Evaluation of the Psycho-Physiological Concomitants of Increasing Alpha-Wave Amplitude in Anxiety and Stress Disorders}

Lesley Parkinson and Alan Parkinson
BrainHealth

\section{Introduction}

Anxiety and anxiety disorders involve both psychological and physiological conditions. Anxiety covers uneasiness, apprehension, fear, and worry in circumstances that do not necessarily warrant it. Accompanying symptoms include those such as heart palpitations, muscle tension, fatigue, headaches, irritability, and sweating. Sufferers can also find themselves overwhelmed by accompanying stressful thoughts/cognitions. Using brainwave oscillations is an important tool for the understanding of cognitive processes. The Alpha Waveband facilitates the integration of brain activity triggered by sensory stimuli and experiential images. It has the best test-retest reliability of all brainwaves, and it is an intraindividual stable trait. It facilitates the organization of a brain-stem network that governs sympathetic nervous system activity. The present study investigated the hypothesis that increasing alpha waveband amplitude through cranial electrotherapy stimulation (CES) would lead to a reduction in the physiological and psychological symptoms of anxiety disorders. CES has been shown to induce the cortex to produce an increase in alpha-wave activity.

\section{Methods}

Sixteen patients complaining of stress or anxiety were recruited. They completed the Depression, Anxiety and Stress Scale. Baseline CNS Measurement of Brainwave activity occurred at the central SMS. Patients were put on a 1 -month waiting list and then reassessed with the same measures as at baseline. They were provided with an Alpha-Stim CES device to use for 2 months for 20 min per day. Posttreatment trial reassessment then occurred.

\section{Results and Discussion}

The measures at each assessment point were analyzed, providing means and significant differences. Key measures were for the AlphaWave amplitudes and the Depression, Anxiety and Stress Scales Scores. Using CES led to a significant increase in alpha-wave activity and a significant reduction in self-report measures of anxiety and stress. The study demonstrated the importance of the alpha wave in reducing the physiological and psychological symptoms of anxiety and stress.

\section{LORETA Phase Reset of the Human Default Network}

Robert Thatcher

\section{Objectives}

The purpose of this study was to compare electroencephalogram (EEG) phase shift and lock durations between different Brodmann areas of the human default mode network. 


\section{Methods}

The EEG was recorded from 19 scalp locations from 70 healthy normal subjects ranging in age from 13 to 20 years. A time series of LORETA current sources were computed from the center voxel of 14 Brodmann areas (BA) comprising the default mode network. The Hilbert transform of the LORETA time series was used to compute the instantaneous phase differences between all pairs of BAs. Phase lock and shift durations were computed for all combinations of BAs in the delta frequency band $(1-4 \mathrm{~Hz})$. Analyses of variance with Bonferroni corrections were computed with main effects of Brodmann areas, hemisphere, and eyes closed versus open conditions.

\section{Results}

Phase shift and lock duration exhibited discrete durations when comparing pairs of BAs. Phase shift and lock durations, whereas discontinuous and unique for each BA, also exhibited exponential changes with distance and were inversely related.

\section{Conclusions}

Each BA is a node in a network that phase locks with other nodes during discrete and specific intervals of time. The results indicate that anatomical nodes behave like a shutter that opens and closes at specific durations giving rise to temporal packets of phase locked clusters of neurons across networks.

\section{Neural Connectivity Theory of Epilepsy}

Robert Coben

Integrated Neuroscience Services

The prevalence of epilepsy in the general population has been estimated to be at least 7 per 1,000 with rates rising over 4 decades (Hauser, Annegers, \& Kurland, 1991). In addition, there are subclinical seizure disorders that lead to unwanted symptoms to conditions such as autism. If one considers all form of potential seizures disorders and their manifestations, the prevalence and societal challenges that these issues cause are tremendous. Although basic neurofeedback (SMR augmentation) has been shown to be effective in reducing seizures (Tan et al., 2009), the duration of treatment is often long (6-12 months) with only an $80 \%$ success rate. Recently, the notion of epileptic neuronal networks has been postulated (Stefan \& Lopes de Silva, 2013). Utilizing such concepts, one can detect connectivity anomalies that trigger seizure events and measure their resolution as well. Data are presented to show how this approach may be used to design potentially more effective neurofeedback treatment plans with greater success rates than previously studied approaches.

\section{Serial Norm-Referenced Quantitative EEG Evaluations During Subacute Recovery from Traumatic Brain Injury: A Validation Study}

Fred Ulam and Charity Shelton

Missouri Rehabilitation Center, University of Missouri Health System

\section{Purpose}

To assess the usefulness of quantitative EEG (qEEG) in tracking recovery of attention/working memory among patients with traumatic brain injuries (TBI) during subacute neurorehabilitation.

\section{Subjects}

Twelve individuals with moderate to severe TBI who were receiving inpatient neurorehabilitation in a university-based hospital and who met inclusion criteria, participated. Twelve carefully matched healthy control subjects also participated.

\section{Methods}

TBI subjects were administered a battery of neuropsychological tests and a quantitative EEG evaluation every 2 weeks, from the time of enrollment to the time of discharge. Here, we focus on the Digit Span test from the Wechsler Adult Intelligence Scale-IV, which includes measures of selective attention and auditory working memory. The neuropsychological tests and the EEGs were obtained within 2 days of one another. Control subjects took the 
same battery of tests and EEGs on two separate occasions, separated by 2 weeks.

\section{Analysis}

Linear regressions were performed for each digit span subtest and relative power within each of the four traditional EEG frequency bands averaged from four cortical regions - left frontal, right frontal, left posterior and right posterior.

\section{Results}

Significant relationships between relative power and digit span were found for patients and controls. For patients, decreases in delta and theta, and increases in alpha accompanied improved performance. Increases in alpha were associated with improvements for controls, while decreases in beta from the left posterior region were associated with improved test scores.

\section{Conclusions}

QEEG measures of relative power were meaningfully associated with performance on tests of attention/working memory for patients and controls. Decreases in slow activity and increases in alpha accompanied recovery of attention following TBI. QEEG appears to be a valid marker of recovery of attentional functions, and may be useful as dependent measure in studies of novel interventions or in guiding neuromodulation treatments.

\section{Loreta $Z$ Score Neurofeedback in the Treatment of Veterans with PTSD and TBI}

Dale Foster

Memphis Integral Neurofeedback Institute

Katherine Veazey-Morris

Veterans Administration Medical Center

War exposes soldiers to multiple traumas, both physiological and psychological. Many veterans return from their tours of duty suffering from both traumatic brain injury (TBI) and posttraumatic stress disorder (PTSD). The complex and idiosyncratic nature of such injuries often results in treatment resistant symptoms. This case series reviews eight cases in which an electroencephalographic normative database functional network symptom checklist match approach to treatment of veterans with both TBI and PTSD was used.

\section{Method}

The 19-channel qEEG data were acquired using Deymed Truscan and analyzed using NeuroGuide and the Thatcher Lifespan Normative database. A symptom checklist match was constructed matching the individual's symptoms with their abnormal functional neural network metrics. Subjects were then trained with LORETA Z Score neurofeedback with the goal of normalizing the EEG power, phase, and coherence metrics that were likely responsible for their symptoms based on the neuroimaging literature. Results were remarkable in most cases with symptoms decreasing up to $10 \%$ per session even in cases when the symptoms had been persistent and stable for years. However, in the cases where the most psychotropic medication was involved, the results were still positive but slower. This functional network, symptom checklist approach to training appears to offer the best available approach to the treatment of complex cases of TBI and PTSD.

\section{The Relation Between Memory Improvement and QEEG Changes in Three Clinical Groups as a Result of EEG Biofeedback Treatment}

Kirtley Thornton

The Neuroscience Center

It is important to understand the relationship between changes in the quantitative EEG (QEEG) variables and memory changes as a result of the EEG biofeedback treatment. With this goal in mind the senior author reviewed his clinical files from the last 5 years and examined the QEEG data addressing relative power and coherence changes and memory (auditory and reading) improvements. The groups involved included (a) normal individuals wanting to improve their cognitive functioning, (b) 
traumatic brain injured (TBI) subjects, and (c) and (d) subjects who can best be classified as having a specific learning disability (SLD). The SLD group was divided between those who are older than 14 tears of age (adults) and those who are younger than age 14 (children) to reference the appropriate age-related normative group values. The analysis revealed significant improvements in auditory and reading memory across all groups as well as changes on the QEEG variables. All of the groups were performing above the normative reference group on measures of auditory and reading memory in terms of percentage differences (24 to 97\%) and standard deviations $(+1.28$ to 1.85$)$. The average auditory memory standard deviation improvement was +1.52 , whereas the average percentage change was $82 \%$. For the reading task, the average memory standard deviation improvement was 1.38, whereas the percentage improvement was $154 \%$. The experimental group was performing 1.66 SD (68\%) above the control group on auditory memory and .90 SD (52\%) above the control group on reading memory measures. For the QEEG variables, the average raw value of the Spectral Correlation Coefficient (SCC) change for alpha was 6.1 points (2.09 SD), for SCC beta1 $(13-32 \mathrm{~Hz})$ 6.53 points (1.81 SD), and for beta2 (32$64 \mathrm{~Hz}) 7.5$ points $(1.77 \mathrm{SD})$. The changes on the relative power measures were less dramatic, albeit significant.

\section{Coherence Training for ASD: Enduring Effects Beyond 3 Years}

Robert Coben

Integrated Neuroscience Services

Follow-up studies of neurofeedback outcome after the active phase of treatment has ended are rare. While some studies have examined outcome 3 to 6 months following treatment (i.e., Gevensleben et al., 2010; Leins et al., 2007), follow-up periods that are longer than this are scant in the literature. Coben, Arns, and Kouijzer (2011) reported on follow-up periods up to 6 months in attention deficit/ hyperactivity disorder and 1 year (Kouijzer et al., 2009) for children with autism spectrum disorders (ASD). Of interest, in the work that has been done to date, it seems that the benefits of therapy are maintained and may even enhance over time. In this talk, I present data from children with ASD treated with neurofeedback including a follow-up at 3 years and a single case study with follow-up period at 8 years. These findings further establish the long-term benefits of neurofeedback.

\section{Efficacy of Cranial Electrotherapy Stimulation for Anxiety, PTSD, Insomnia and Depression: Military Service Members and Veterans Self Report}

\section{Jeff Marksberry \\ Electromedical Products}

Cranial electrotherapy stimulation (CES) is prescribed for service members and veterans as a complementary or alternative medicine modality for the treatment of anxiety, posttraumatic stress disorder (PTSD), insomnia and depression. The purpose of this study was to examine service members' and veterans' perceptions of the effectiveness and safety of CES treatment. service members and veterans $(N=1,514)$ who had obtained a CES device through the Department of Defense or Veterans Affairs Medical Center from 2006 to 2011 were invited to participate in the webbased survey via e-mail. One hundred fifty-two participants returned questionnaires. Data were analyzed using descriptive statistics. The majority of participants reported substantial clinical improvement $(>50 \%)$ from using CES: anxiety $(66.7 \%)$, PTSD $(62.5 \%)$, insomnia $(65.3 \%)$, and depression (53.9\%). Respondents also perceived CES to be safe $(99.0 \%)$. Those individuals who were not taking any prescription medication rated CES as more effective than the combined CES and prescription medication group. CES provides service members and veterans with a safe, noninvasive, nondrug, easy-to-use treatment for anxiety, PTSD, insomnia, and depression that can be used in the clinical setting or self-directed at home. 


\section{Neurofeedback for Early Psychosis: A Theoretical Rationale for Cognitive Rehabilitation}

Thomas Cothran, Jonathon Larson, Lindsay Sheehan, Bethany Apa, and Christopher Haak Illinois Institute of Technology

Cognitive impairments in schizophrenia are the strongest predictor of functional outcomes, are relatively unaffected by current pharmacological interventions, and are present prior to the first psychotic episode. Deficits in cognitive control appear to be a core characteristic of schizophrenia. Cognitive control refers to processes that allow information processing and behavior to vary adaptively in the moment. The construct encompasses a broad range of mental operations including working memory, contextual processing, and attention allocation. It appears related to a general-purpose, superordinate network with critical nodes in the dorsolateral prefrontal cortex (DLPFC), anterior cingulate cortex (ACC), and mediodorsal nucleus of the thalamus (MDN). Recent evidence suggests that impairments in cognitive control result from disruption in the coordination of activity across brain regions that may stem from deficits in the development of normal brain connectivity. Abnormalities in neural synchrony are present in schizophrenia. Basic research suggests that deficits in neural synchrony underlie the development of deficient connectivity within and between distributed neural networks. During adolescent brain maturation a shift occurs from local to global coordination of brain states. In schizophrenia, nascent connectivity issues during childhood, that may be limited to small-world networks, are then unable to support the shift to higher order organization, resulting in disability. Precise neural synchronization between neural assemblies appears to promote longterm potentiation and the development of cortical networks. The absence of such synchronization may produce depression in the connection between neurons. Deficits in synchrony could plausibly explain the specific dysfunction between DLPFC, ACC, and MDN that underlies impairments in cognitive control. This presentation will review neurofeedback studies for schizophrenia and a neurodevelopmental model of the disorder. Finally, a rationale for the use of neurofeedback in treating cognitive impairments in early psychosis within the context of a clinical staging model will be discussed.

\section{Neuromodulation of EEG LORETA Connectivity Measures to Support Abstinence From Alcohol and Drugs}

Jack Johnstone

Q-Metrx, Inc.

George Fein

Neurobehavioral Research, Inc.

Alcohol dependence is a disorder with an impulsive and compulsive drive toward alcohol consumption and an inability to control or inhibit alcohol consumption. Neuroimaging studies suggest that these behavioral components correspond to an increased involvement of regions that mediate appetitive drive and reduced involvement of regions that mediate executive inhibitory control. We have recently shown using functional connectivity fMRI that, compared to nonsubstance abusing controls, long-term abstinent alcoholics evidence (a) decreased synchrony of limbic reward regions (e.g., caudate and thalamus) with both the Anterior Cingulate Cortex (ACC) and the Nucleus Acumbens (NAcc) and (b) increased synchrony of executive control regions (e.g., dorsolateral prefrontal cortex) with both the NAcC and the subgenual ACC. These results are graded with duration of abstinence and are present both in cross-sectional and longitudinal studies. These results are consistent with a compensatory mechanism that develops with abstinence such that at rest, decisionmaking networks are primed to be less responsive to appetitive drive stimuli and ready to exhibit inhibitory control. Facilitation of these adaptive changes may show promise as a treatment for individuals pursuing abstinence from alcohol and drugs. Studies to be reported here 
are extending these findings from $\mathrm{FMRI}$ to EEG using LORETA connectivity analysis with the goal of developing a neurofeedback treatment supporting long-term abstinence from alcohol and drugs.

\section{Continuous Performance Test Results Following Neurofeedback and the Efficacy of Frequency Optimization Using Bipolar Training Montages}

John Putman

The EEG Institute

The existence of Infra low (or Very Slow) frequencies in the brain has been known for many years, but their relevance to the EEG has only recently begun to be understood. (Very Slow frequencies are defined as those $0.1-0.01$ or lower). Although their origin is not completely clear, evidence suggests that they play a fundamental role in the management of cortical dynamics. These low-frequency oscillations also play a role in attention where lower amplitudes of infralow activity are correlated with attention deficits (Helps et al., 2007). In addition, shifts in slow cortical potentials seem to precede the onset of seizures (O'Leary \& Goldring, 2007).

\section{Neurotherapy for Sustainable Addiction Recovery: An Integrated Model}

Judith Ann Miller

International Neural Renewal/Courage to Change Ranch

Three decades of research and development call for a state-of-the-science addiction recovery model. This paper introduces An Integrated Solution for Addiction Recovery. The purpose of this paper is threefold: (a) to present a historical perspective on the advent of neuroscience adapted for addiction treatment, (b) to present an analytical case series report that reveals neuro-therapy to be a promising therapy for addiction solutions, and (c) to present an Integrated Model for Sustainable Addiction Recovery. The research reported in this paper is based on a 2-year progress report (2011 to 2012) with a total sample size of 100 and (2012-2013) with a total sample size of 100 addicted clients of an addiction recovery program. Following neurotherapy, $100 \%$ of the 200 clients experienced profound relief from the symptoms of addiction (depression, anxiety, insomnia, PTSD, and dual diagnosis) suffered prior to treatment.

\section{Exercise for Attention: QEEG, ERP, and Behavioral Evidence}

\section{Lindsay Thornton \\ USOC \\ Alex Thornton}

The link between exercise and cognition has been established (Chang et al., 2012; Smith et al., 2010). Effects tend to be small but significant, and tend to occur in executive function, memory, and attention domains. Although there have been many studies that have examined cognitive function in adults, purely cognitive studies of exercise and cognition in children are rare (Best, 2010). There are many studies of children in the context of academic performance. A review of school studies is contained in a recent CDC (2010) white paper; exercise in school generally has a positive effect or no effect on academic and cognitive performance. Attention deficit/hyperactivity disorder (ADHD) specific research on exercise is scarce. Archer and Kostrzewa (2012) suggested that ADHD may be improved by exercise, noting that ADHD individuals tend to have deficits in many areas that are improved by exercise (Halperin \& Healey, 2011). Preliminary evidence suggests that ADHD children may demonstrate improved executive function after a long-term exercise program (Gapin et al., 2011). A recent study (Pontifex et al., 2013) has demonstrated positive effects of a single bout of exercise on task switching and the P3 component in ADHD, building on previous work (Pontifex et al., 2011) showing differences in P3 latency and amplitude across high-fit and low-fit children. In the present study, students 
$(N=55)$ were recruited to participate in a month-long, before-school cycling program. Twenty-two unmedicated students had an ADHD diagnosis or had reported attentional difficulties and participated in QEEG and ERPs at pre- and postmeasure. Decreases in theta, $\mathrm{mu}$, and alpha were observed at various sites across the cortex. P3b latency and amplitude improved. Although the sample size is small, this study provides evidence of neuroeletric changes toward the norm in unmedicated children with attention deficits at baseline, and improved attentional behaviors.

\section{The Impact of Audio Environment on Attention}

\section{Charles Wasserman}

This study examined the relationship between audio environment and attention. Although background noise has generally been assumed to be distracting, recent research has suggested that the opposite may be true. Attention was assessed in 27 participants using a continuous performance test under three different noise conditions: exposure to ambient noise (the control), pink noise, and an audio track from television. Attention was significantly improved in pink noise as compared to the ambient noise, whereas no differences were found between ambient and television conditions. These findings suggest that not all noise is created equal when it comes to paying attention.

\section{Using Neurofeedback in a Forward Operating Base: Afghanistan July 2011-July 2012}

Michael Villanueva US Army Medical Service Corps 7214 Medical Support Unit

Colin Rader

US Army 504th BFSB

This presentation discusses lessons earned from implementing neurofeedback within an Afghanistan Forward Operating Base. Four perspectives are addressed: Clinical, Combat
Operations, Program, and Medical. Clinically, neurofeedback proved effective in (a) rapidly relieving operational stress symptoms; (b) facilitating personal management of Axis I and II symptoms and behaviors, including easing signature $\mathrm{mTBI}$ symptoms; and (c) reducing recovery time from strenuous physical training. Operationally, neurofeedback delivered both unanticipated and unexpected benefits: Senior Officers used Alpha Theta (AT) sessions to facilitate visualization in mission planning, Intelligence Officers used AT to scaffold perceptual abilities, and patrol leaders used AT and Infralow neurofeedback sessions to facilitate adjustment from long patrol hours noting less distractibility, less irritation, and less fatigue - overall, a rapid return to "baseline" after kinetic patrols. Programmatically, clinic utilization rates of neurofeedback by soldiers over the course of the 12-month deployment suggests the neurofeedback "training the brain" paradigm to be congruent with Army values in that sessions were viewed as a metric of personal responsibility, a training tool, rather than a behavioral health intervention. This perception may have been key in reducing stigma commonly associated with accessing behavioral health services. Medically, the Battalion Aid Station observed a decline in the number of prescriptions issued for sleep and anxiety, increased mood stabilization in soldiers, and increased cognitive efficiency in key infantry units. Effectiveness of neurofeedback engendered a closer working relationships with army and navy doctors and psychiatrists who monitored psychiatric medications in person or vis-à-vis telemedicine. Overall, anecdotal evidence from deployment suggests neurofeedback demonstrates cultural sensitivity to a special population's operational and cultural needs.

\section{Breathing, Blood Flow, and the Brain- An Evolutionary Understanding}

\section{Stephen Elliott \\ COHERENCE LLC}

Resonant breathing generates a blood wave in the circulatory system of significant magnitude. 
This wave rises in the arterial tree during exhalation and rises in the venous tree during inhalation. During exhalation, oxygenated blood exits the lungs, flows through the left heart and into both ascending and descending aortas, the ascending aorta supplying the head and brain. The physiological impetus for the wave is movement of the diaphragm. This presentation asks, "What is the evolutionary necessity for the wave and for the diaphragmatic action that produces it?" An argument is presented that the diaphragm is necessary to move blood upward against gravity and evolution of the diaphragm correlates with physical erectness of vertebrate life and of man.

\section{Clinical Use of Delta Peak Frequency Neurofeedback for Mild Head Trauma}

Frank Morganti

Pittsburgh Hyperbaric Institute

Richard Genardi

Private practice

This talk presents assessment data and clinical results obtained by using the peak frequency of Delta $(1-4 \mathrm{~Hz})$ as a key indicator and neurofeedback parameter. This approach made it possible to obtain improvements in clients who did not respond to previous interventions. QEEGs were inspected of patients who had suffered one or more concussions and participated in a hospital based concussion program but failed to remit their symptoms back to normal, even after 20 or more hyperbaric oxygen treatments were given after the concussion medical treatment failed. QEEGs were given pre- and posthyperbaric treatment and after medical failure, and further inspection of qEEGs results revealed unchanged, significantly elevated Delta peak frequency in all or a majority of the 10/20 sites acquired during the pre and post qEEGs in the range of $(2.0-2.8 \mathrm{~Hz})$. These patients varied in age from 13 to 19 years old. Several other older adults that had presenting symptoms of slow processing, depression, and short-term memory symptoms and were medially treated specifically for those symptoms underwent qEEG. They also had elevated Delta peak frequency, and when questioned, remembered having $\mathrm{mTBI}$ years before that was undiagnosed. Their symptoms were tracked across multiple brain functional sites by a rating scale linked to fMRI findings to that site. Diffuse axonal injury was evident by involvement of a majority dysfunctioning sites. As a result of this finding, a neurofeedback protocol was developed to reward reduction of Delta peak frequency between 1.7 and $1.9 \mathrm{~Hz}$. Patients demonstrated within-session changes of $0.5 \mathrm{~Hz}$ or more in Delta peak frequency, demonstrating effective operant training to reduce this frequency to enter the normal range.

\section{Immediate and Follow-Up Success of Neurofeedback Treatment for 20 Severely Depressed Clients: Quadrant Brain Theory and Application}

\section{Angelo Bolea and Dennis Romig \\ Private practice}

The application of the quadrant brain theory was previously demonstrated as successful in the neurofeedback treatment of chronic inpatient schizophrenia (Bolea, 2010). The quadrant brain theory and rationale was since applied with a cohort of 20 suicidal and severely depressed physician-referred patients. The initial treatment success was $95 \%$ with only one client dropping out of treatment. The 12-month follow-up had $0 \%$ relapse in all clients that completed treatment. These results compared to $50 \%$ relapse for prescription drug only treatment for depression study groups. The quadrant brain theory and methodology's utility may extend beyond success in treating depression and schizophrenia. The theory is presented as well as its application in the treatment of depression.

\section{Two-Year Pilot Study of Neurotherapy and Audiovisual Entrainment in a Private, RuraL K-12 School}

Rebecca Ruefer, Shirley Dyk, and Paul Swingle

A 2-year study evaluating the feasibility of offering neurotherapy and audiovisual entrainment 
in a private $\mathrm{K}-12$ school in rural Montana began in August 2011. Several groups of students were included, comprising both mainstream neurotypical students and resource students and ranging in age from 6 to 18 years. All students were evaluated using the Clinical $Q$ assessment prior to treatment and again every 10 sessions. The Clinical $Q$ reports were e-mailed to a long-distance mentor, and the appropriate protocols were e-mailed back to the providers. The providers were a bioengineer and a special education teacher, both of whom had undergone training but neither of whom was BCIAcertified. The logistics, feasibility and results, both behavioral and academic, are presented.

\section{Integral Neurofeedback is Superior to Conventional Medicine in a Case of Bipolar Disorder}

\section{Dale Foster}

Memphis Integral Neurofeedback Institute

Bryan Butler

Memphis Integral Neurofeedback Institute

Wes Center

Focus for Living

Conventional medicine views bipolar disorder as a biochemical disease with a psychopharmacological treatment but no cure. Although this perspective offers some utility, recent advances in neuroscience offer alternative models that are more effective in improving the function and quality of life of those who suffer from this illness. This 20-year case study of a patient diagnosed with bipolar disorder is viewed through the lens of the Integral AQAL model, including linear and nonlinear systems perspectives as well as subjective and intersubjective factors. The patient's recovery and development is illustrated through surface qEEG, LORETA, and SPECT neuroimaging, subjective report, and neuropsychological assessment as he progressed from physical disability to a highly functioning adult via an integral neurofeedback approach. Using multiple modalities of neurofeedback, biofeedback, neuro-modulation, neuro-stimulation, lifestyle management, neurochemistry management, and intersubjective experiential work, this individual was able to decrease psychotropic medication from six prescriptions to zero, overcome numerous posttraumatic stress symptoms, and improve cognitive function to the point of completing a graduate degree and becoming a licensed health service provider. The typical prognosis of a patient with bipolar disorder treated with conventional medicine is compared with the superior possibilities of an integral neurofeedback approach based on this case study.

\section{Neurofeedback in a Public School Setting: Efficacy as an Intervention for ADHD, Inattentive Subtype to Improve Reading Achievement}

\author{
Jeffry La Marca \\ University of California, Riverside
}

Neurofeedback has been used since the 1970s as an intervention to address the symptoms of attention deficit disorder/attention deficity hyperactivity disorder (ADD/ADHD). Studies consistently suggest that neurofeedback training enhances cognitive performance (Vernon et al., 2003), increases IQ scores (Linden, Habib, \& Radojevic, 1996), and improves attention (Leins et al., 2007). Furthermore, research indicates that neurofeedback is most efficacious for ameliorating symptoms of inattention, which are associated with learning difficulties and academic problems. Addressing the needs of students with ADD/ADHD is especially critical in schools, as this is where most children are first identified and their impairments become evident (USDE, OSERS, \& OSEP, 2008). Research consistently demonstrates that attention deficits have a deleterious effect on academic attainment (Barkley, 2002). Children with ADHD, inattentive subtype have considerably more problems with processing speed than both typically developing peers and students with other subtypes (Ghelani, Sidhu, Jain, \& Tannock, 2004). Studies have also found that individuals with the inattentive subtype process visual information slowly and exhibit impairments in allocating attention to information within their visual field (Swanson, 
Posner, Potkin, \& Bonforte, 1991). Reading and math disorders, along with other learning disabilities, appear to be more prevalent in individuals with the inattentive subtype than found in those with the predominately hyperactive-impulsive type (Willcutt \& Pennington, 2000). Although medical and psychological interventions cannot be ignored, especially because these are often implemented with the specific goal of maximizing school success, the responsibility for accommodating students with special needs in school ultimately falls to educators. Neurofeedback may have the potential to be used by highly trained educators to improve school performance. This presentation discusses the findings of the first study to directly examine the use of neurofeedback as an intervention to improve attention, reading fluency, and reading comprehension in a public school setting.

\section{Modeling EEG-Band Neurofeedback: Modulating Internal States Without Conditioning of EEG Sources}

Leonardo Trejo

Pacific Development and Technology, LLC

Roman Rosipal, Nayson Fernandes, and Rasheed Akbarut

At least three neurofeedback techniques can produce measurable behavioral and neurophysiological changes. These involve modulating slow-cortical potentials (SCP), fMRI BOLD signals, or amplitude (or power) in narrow EEG bands. We think SCP neurofeedback may work indirectly by conditioning task-specific attentional focus, whereas fMRI neurofeedback directly conditions regional brain activity during task performance. However, the object of conditioning in EEG-band training is unclear because there is no overt task to perform, and the electrophysiological and behavioral changes can occur rapidly. In a previous double-blind, placebo-controlled experiment, we trained participants to increase EEG amplitude in narrow bands (C3-SMR, C4-SMR, C3-Beta, sham) using auditory rewards and 30-min training sessions over 5 consecutive days. We observed widespread changes in ERP/ERSP patterns for reward stimuli during training. However, we did not find significant conditioning of trained bands within or across sessions. We also observed improved performance of a hemispheric attention task in the C3-Beta group after five sessions. To explain these and related results we are developing a four-part model: First, introspective tasks, such as mind wandering during rest or autobiographical memory, engage a Switching Network that activates an internally directed Default Mode Network and deactivates externally-directed problem-solving networks, Second, the DMN activates a "self-control" system that links rewards to internal states of arousal, motor activation, attentional focus, or cognitive engagement. Third, the internal states covary with synchrony in fundamental EEG "atoms." We separately developed EEG atom metrics, where each atom represents one oscillatory EEG mode in the $1-40 \mathrm{~Hz}$ range, with a unique spectral envelope, topographical power distribution, and pattern of connectivity. Fourth, when the self-control system is engaged, neurofeedback rewards condition internal state modulations or "responses" that cause EEG atom synchrony levels to change accordingly. Thus EEG-band training conditions the self-control system, which can then manage internal states and global network connectivity.

\section{The Influence of Individual Alpha Peak Frequency on Resting State EEG in Adults With Attention-Deficit/Hyperactivity Disorder}

Sarah Wyckoff

University of Tuebingen/University of Pennsylvania

\section{Objectives}

Adult attention-deficit/hyperactivity disorder (ADHD) has been characterized by deviant patterns of EEG activity during resting state, particularly increased theta and decreased beta, to a lesser degree. A recent investigation of childhood ADHD populations indicated that theta/beta activity was mediated by individual differences in alpha peak frequency (iAPF). The present 
study seeks to investigate whether iAPF influence EEG findings in adults with ADHD, as well as to explore the relation between resting-state brain oscillations, iAPF, and core ADHD behaviors.

\section{Methods}

Continuous 21-channel EEG was acquired from 46 adult participants with DSM-IV defined ADHD and 46 healthy controls. For each frequency band (delta, theta, alpha, beta, theta/ alpha, and theta/beta power), power analysis (absolute and relative power), and condition (eyes-closed and eyes-open), a mixed analysis of variance was used to examine the effects of region and group using fixed frequency and individualized IAPF bands. Partial correlation coefficients were calculated between ADHD behavioral measures and QEEG data.

\section{Results}

Using fixed frequency bands, ADHD participants presented with elevated theta, beta, theta/alpha, and attenuated alpha activity. However, several interactions within the theta, beta, and theta/ alpha frequency bands were no longer significant after using individualized frequency bands. No consistent relation was found between restingstate brain oscillations and ADHD behaviors, with the exception of a weak positive correlation between iAPF and inattention. The present results suggest that discrepant QEEG findings within adult ADHD research may reflect differences in iAPF. In addition, EEG activity in adult ADHD appears to be mediated by distinct neurophysiological subgroups such as frontal theta and high alpha peak frequencies.

\section{Conclusion}

Future research should investigate the functional role of resting-state brain oscillations by investigating neurophysiological subgroups and controlling for iAPF.

\section{The Pause That Reinforces: The Role of Arousal Modulation in EEG Operant Conditioning}

M. Barry Sterman

David Geffen School of Medicine, UCLA
Currently, the field of neuroscience is driven mainly by genetics, neurochemistry, cell biology, and technology-enabled brain imaging. In an earlier age, the field was more focused on neuro-physiology and system organization. This presentation attempts to integrate these two approaches in proposing a rational model for the processes that enable learning in general and operant conditioning in particular. Specifically it focuses on reward. What system organization produces changes in brain physiology and circuit dynamics as a result of reward? How do these changes produce response reinforcement? What neurofeedback methodologies actually promote and confirm these events?

\section{Neurotherapy for the Treatment of Noncombat Posttraumatic Stress Disorder: A Case Report}

\section{Corey Feinberg and Elsa Baehr}

NeuroQuest

This is the case report of a female adult diagnosed with posttraumatic stress disorder (PTSD) that was not induced by combat or trauma experienced in the line of duty, but rather the posttraumatic psychological distress of having been reared by a mother who was a survivor of the Holocaust. The purpose of this report is to examine the subject's neurological and behavioral response to neurofeedback training as a treatment intervention for her symptoms of PTSD. Pretreatment baseline measurements were taken that included an initial intake interview where the subject described the nature and severity of her symptoms, a Quantitative EEG analysis was performed, and self-report inventories were administered using Beck short forms for depression (BDI) and anxiety (BAI). A customized neurotherapy treatment strategy was applied using QEEG guided neurofeedback protocols involving posterior, eyes-closed combinations of Live Z-Score Training for power and coherence, as well as traditional amplitude training protocols. Client observations were recorded at various significant points to reflect self-reported changes in 
symptoms, and professional observations were gathered about treatment progress from the patient's therapist. In this case, the neurotherapy treatment strategy was able to produce significant changes in the subject's EEG toward a pattern of normalization. These changes in neurology mirrored positive patterns of remediation in the subject's symptoms of PTSD that showed a significant reduction in both severity and frequency of occurrence. The improvements in behavior were observed by the clinician administering treatment, the subject's therapist, and by the patient herself. Posttreatment assessment indicated decreases in anxiety as measured by the BAI and qualitative improvements in the post QEEG.

\section{LORETA Z-Score Neurofeedback as a Potential Application in Depression and Anxiety}

J. Lucas Koberda, Andrew Bienkiewicz, Andrew Moses, Laura Koberda, and Paula Koberda Tallahassee Neurobalance Center

Prior reports have already demonstrated that standard 1-2 electrode neurofeedback (NFB) is beneficial in the treatment of depression and anxiety (Dias, 2011; Hammond, 2005). Recent developments in computer technology and the introduction of LORETA NFB enable more precisely targeted therapy. This report contains analysis of 31 patients who were enrolled in LORETA Z-score NFB due to depression and anxiety. Many of the patients were referred for NFB by psychiatrists due to resistance of the symptoms to medications. A patient's workup included EEG, QEEG/LORETA electrical imaging as well as brain imaging. LORETA showed areas of frontal lobe or Anterior Cingulate dysregulation as the most frequent finding. The design of this study was to complete a QEEG before beginning NFB to conduct QEEG-guided biofeedback treatment. A QEEG was also completed after 10 sessions of NFB therapy to see if any objective improvement in QEEG/LORETA abnormalities was noted. Patients were also requested to give their subjective feedback on the degree of symptoms improvement. Most of the patients completed at least 10 sessions of NFB, and the degree of subjective improvement (if any) was recorded. Twenty-four patients $(77 \%)$ were found to have subjective and/or objective improvement. These results are very promising, and indicate high potential effectiveness of LORETA Z-score NFB in the treatment of anxiety and depression.

\section{Coherence: Toward Multivariate Analyses and Training}

Robert Coben

Integrated Neuroscience Services

Traditionally and historically, EEG coherence estimates have arisen from cross-correlations between pairs of electrodes (Bendat \& Piersol, 1980; Otnes \& Enochson, 1972). These concepts have commonly been used and applied. In fact, a search in Google Scholar for "EEG coherence pairs" revealed more than 14,500 citations. Concerns in the literature have emerged about the accuracy of pairwise measures (Barry, Clarke, McCarthy, \& Selikowitz, 2005), and research has demonstrated that multivariate methods more accurately reflect the true nature of connectivity (Blinowska, 2011). These findings demonstrate the need for multivariate strategies for assessing coherence. These various methods are presented and discussed during this talk. Given that multivariate assessment strategies lead to more accurate coherence measures, it only makes sense that multivariate coherence training methods should be explored as well. One such initial approach is also presented.

\section{Neurofeedback as an Alternative Treatment for Chronic Primary Insomnia}

Olivier Pallanca

Paris Hospital APHP-IMC

Yann Renard

The pathophysiology of Primary Insomnia (PI) remains mostly unclear, but cortical hyperarousal may represent a final common pathway in the development and maintenance of the 
disorder, which might cause the experience of sleep onset insomnia and non-restorative sleep (Perlis, 1997; Riemann, 2010). Several studies have shown that patients with insomnia exhibit elevated levels of Beta EEG activity $(14-35 \mathrm{~Hz})$ at or around sleep onset and during NREM sleep and EEG spectral power in the beta range has been suggested to be an index of cortical arousal (Buysse, 2008; Freedman, 1986; Merica, 1992; Perlis, 2001). Furthermore, cognitive behavioural therapy for insomnia, the first-line treatment for primary insomnia, reduces beta activity in insomnia patients (Cervena, 2004). A few studies involve neurofeedback as a treatment for primary insomnia, and most of the studies that use the SMR techniques show improvement in sleep and daytime functioning (Cortoos, 2006; Hammer, 2011). Methodology and preliminary results: The aim of our study is to better characterize the cortical hyperarousal that is exhibited by patients with $\mathrm{Pl}$, especially during periods of wakefulness and during sleep onset, in comparison with a control group. The two groups (15 PI and 15 controls) were recruited after the validation of the $\mathrm{Pl}$ diagnosis using the International Classification Sleep Disorders 2 criteria. The EEG was recorded with a Nexus 32 (Mindmedia) with a 21-electrode EEG cap, with concomitant additional channels for heart rate and skin conductance in order to correlate the emotional and EEG changes. The Openvibe qEEG technique was used to analyze the signal. In preliminary analyses, the Beta EEG activity seems to be more elevated in some cortical areas in the PI group. If the results are confirmed, we want to develop a special neurofeedback technique to decrease the cortical hyperarousal during wakefulness in patients with PI.

\section{Toxicity and Nutrition-Effects on Nervous System Function}

\section{Patricia Ryan}

Alternatives-A Center For Conscious Health

Practitioners are aware of the benefits of neurofeedback for a variety of disorders including attention deficit/hyperactivity disorder, anxiety, depression, and insomnia. The purpose of this presentation is to expose the practitioner to the data behind neurotoxicity, nutrition, and brain dysfunction. See how genetically modified foods and herbicides cause physiologic disruption. Many chemicals including heavy metals, solvents, and aspartame are causing damage to our neurons. In addition, information about food allergies and Candida overgrowth and their link to symptoms such as brain fog and irritability, and cognitive dysfunction is discussed. Some nutritional pearls are given to the practitioner as well.

\section{Burnout, Interpersonal Commitment, Client Adherence, Continuing Education, and Quality of Work Life Among Neurofeedback Practitioners}

Jonathon Larson, Thomas Cothran, Lindsay Sheehan, Katherine Kereszturi, Bethany Apa, Catherine Ryan, Robert Beedle, Christopher Haak, and Kelly O'Neill

Illinois Institute of Technology

This presentation discusses a study of neurofeedback (NFB) practitioner self-perceptions related to quality of work life. Practitioner self-perceptions of common clinician factors related to NFB were identified. To guide this current study, we utilized our previous conceptual framework research on practitioner perspectives of NFB. One hundred forty-eight NFB practitioners completed online surveys gathering demographic information and ratings of practice behaviors and characteristics. Our results indicated that $74 \%$ of the variance in quality of work life can be explained by a significant multiple correlation of burnout, interpersonal skills commitment, and client adherence. We found monthly sessions correlated with financial gain or loss (FGL). We also found client adherence separately correlated with monthly sessions, NFB knowledge, NFB learning commitment, and NFB mentorship. For NFB practitioner self-perceptions of common clinician factors, the most frequently endorsed practitioner traits in rank order were (a) ethical, (b) attentive, (c) empathic, (d) calm, (d) observant, (e) sense of humor, (f) analytical 
and confident (tied), (g) friendly and realistic expectations (tied), (h) optimistic, and (i) careful. NFB practitioner quality of work life appeared to be related to three straightforward components: reducing burnout, increasing commitment to enhancing interpersonal skills, and increasing client adherence. Practitioners providing mentoring, practitioners improving NFB knowledge and skills, and more monthly sessions are separately related to client adherence. Of interest, we found the number of monthly sessions positively correlated with monthly FGL. We found a variety of perceived NFB common clinician factors adding to the complexity of understanding factors influencing NFB outcomes. Of interest, two (attentive and calm) of the top four practitioner self-perceptions of common clinician factors are also important NFB client outcomes. Finally, a post hoc analysis looking at groups with differing levels of monthly continuing education hours found significantly higher net income among practitioners with higher average hours.

\section{Cumulative Effects of Anodal tDCS on EEG Oscillations and Attention Regulation Among Individuals With Traumatic Brain Injury}

\section{Fred Ulam}

Missouri Rehabilitation Center, University of Missouri Health System

Ben Hunter

Forest Institute of Professional Psychology

\section{Purpose}

The purpose of this study is to explore the effects of transcranial direct current stimulation (tDCS) on EEG oscillations and attention regulation among individuals with traumatic brain injuries (TBI) undergoing inpatient neurorehabilitation.

\section{Subjects}

Twenty-six individuals with moderate to severe TBls participated in a randomized, placebocontrolled, double-blind study of tDCS, with 13 in the active and 13 in the sham groups.

\section{Methods}

Anodal stimulation to the left dorsolateral prefrontal cortex (F3), with the cathode over the right supraorbital area (Fp2) was provided for $20 \mathrm{~min}$ at $1 \mathrm{~mA}$ over 10 daily sessions. Dependent measures included relative power derived from six quantitative EEG studies obtained 2 days prior to the first tDCS session, immediately before and after the first session, immediately before and after the 10th session, and 2 days following the 10th session. A battery of neuropsychological tests assessing aspects of attention/working memory, inhibitory control and cognitive flexibility were administered before the first and after the 10th tDCS treatment.

\section{Analysis}

A repeated measures analysis of variance with post hoc $t$ tests was used to examine EEG and neuropsychological differences.

\section{Results}

For the active tDCS group, theta decreased immediately following the first session at F3. Decreased delta was seen for the active group between the first $q E E G$ and the last, at Fp2. Increases in alpha were seen for the active group at both F3 and Fp2 between the first qEEG and the final posttreatment qEEG. No significant EEG changes were seen for shams. The active group with EEG slowing at the outset showed significant improvement in seven of nine neuropsychological tests, whereas the active group without slowing improved on only one test. Shams with slowing improved on three of nine tests, as did shams without slowing.

\section{Conclusions}

This preliminary, exploratory study suggests tDCS can safely modulate cortical excitability among victims of $\mathrm{TBI}$ with beneficial effects on attention regulation.

\section{Concussion Hits Hard: Recovery from the Multiple Effects of Concussion: Requires Interventions Be Based on an Appropriate Multimodal Assessment}

Lynda Thompson and Michael Thompson 
ADD Centre and Biofeedback Institute of Toronto

Assessment of the patient who has suffered a concussion should first be carried out by a knowledgeable medical specialist. However, MRI, PET, and CAT scans often show no abnormality. In addition, traditional rehabilitation often meets the criteria of conservative "do no harm" but may do little to remedy the deficiencies caused by minimal-to-moderate injury. This presentation suggests a methodology for efficient and accurate assessment that can lead to effective intervention. The EEG and evoked potentials (ERP) can reveal the effects of damage elicited by stretching and twisting of axons, called diffuse axonal injury (DAI). The evoked potentials are particularly important for reflecting brain speed. Concussion may injure the right and/or left insula. This will impair heart rate variability. Thus, concussion assessment should measure QEEG, ERPs, $H R V$, and, using continuous performance tests, measure attention, impulsivity, and variability of response time. This should be combined with neuropsychological testing, in particular for short- and long-term memory, attention span, impulsivity and questionnaires regarding medical health, depression, and anxiety. When available, balance assessment of vestibular function with a "force-plate" can be helpful. Extended biochemical assessment can be added, and this can lead to appropriate dietary and supplement interventions. This presentation reviews some of the literature on TBI and the connection to cardiac problems. It then outlines the neuroanatomical underpinnings regarding why, how, and with what effect LORETA--score NFB can be used in conjunction with some combination of HRV training, transcranial direct current stimulation, passive infrared feedback, and metacognitive strategies, in addition to dietary interventions, to bring a client back to high-level functioning. The theoretical aspects of this presentation will be supported by case examples: a PhD candidate in artificial intelligence, an author, a graduate student in finance, and an athlete. 